\title{
Isolating strong-field dynamics in molecular systems
}

\author{
Gal Orenstein, ${ }^{1, *}$ Oren Pedatzur, ${ }^{1, \dagger}$ Ayelet J. Uzan, ${ }^{1}$ Barry D. Bruner, ${ }^{1}$ Yann Mairesse, ${ }^{2}$ and Nirit Dudovich ${ }^{1}$ \\ ${ }^{1}$ Department of Physics of Complex Systems, Weizmann Institute of Science, 76100, Rehovot, Israel \\ ${ }^{2}$ Université de Bordeaux, CNRS, CEA, CELIA, UMR5107, F33405 Talence, France
}

\begin{abstract}
Strong-field ionization followed by recollision provides a unique pump-probe measurement which reveals a range of electronic processes, combining sub-Angstrom spatial and attosecond temporal resolution. A major limitation of this approach is imposed by the coupling between the spatial and temporal degrees of freedom. In this paper we focus on the study of high harmonic generation and demonstrate the ability to isolate the internal dynamics-decoupling the temporal information from the spatial one. By applying an in situ approach we reveal the universality of the intrinsic pump-probe measurement and establish its validity in molecular systems. When several orbitals are involved we identify the fingerprint of the transition from the single-channel case into the multiple-channel dynamics, where complex multielectron phenomena are expected to be observed.
\end{abstract}

Attosecond science has revolutionized our ability to capture electron dynamics on extremely short time scales. These dynamics are often observed by applying an attosecond pulse as a pump or a probe of a fast-evolving process $[1,2]$. An alternative approach-high harmonic generation (HHG) spectroscopyuses the attosecond pulse generation mechanism itself as an extremely accurate time-resolved measurement [3]. In this self-probing scheme the strong-field tunnel ionization acts as a pump, removing an electron from an atomic or molecular system, thus initiating a hole wave packet. Driven by the laser field, the liberated electron returns to the parent ion and probes it via radiative recombination, leading to the emission of high-order harmonics of the driving laser field [4,5]. These measurements combine Angstrom spatial resolution, provided by the de Broglie wavelength of the returning electron, with attosecond temporal resolution provided by the ionization and recollision dynamics [3,6].

The heart of HHG spectroscopy lies in the link between the pump (ionization) and the probe (recollision), defined by electron trajectories driven by a strong laser field [4,5,7]. This link is assumed to be universal and dictated by the ponderomotive energy $U_{p}$ and ionization potential $I_{p}$. However, so far such a link has been measured and verified for simple atomic systems only [8-12]. Long-term perspectives of HHG spectroscopy aim at resolving attosecond-scale phenomena in complex molecular systems, where nontrivial multielectron interactions evolve. In such systems the interaction with the laser field commonly involves the contribution of several molecular orbitals (channels), leading to population transfer or electron rearrangement [13-19]. The application of HHG spectroscopy as a state-of-the-art spectroscopic tool raises the following question: Can we extend the role of the internal pump-probe measurement to molecular systems and identify its limitations?

Extracting the temporal information encoded in the HHG spectrum is a primary challenge. Two main approaches have addressed this challenge: the ex situ and the in situ measurement schemes. The first approach, reconstruction of

\footnotetext{
*gal.orenstein@weizmann.ac.il

†orenpedatzur@gmail.com
}

attosecond beating by interference of two-photon transitions (RABITT) [20], resolves the spectral phase of the emitted attosecond pulses in an external gas target. An alternative in situ measurement probes the dynamics of electron trajectories by perturbing the harmonic generation mechanism itself using a weak second harmonic (SH) field [10,21]. While both approaches have been applied to characterize attosecondscale dynamics, they are fundamentally different. A thorough experimental comparison studied their macroscopic difference [22]. Recently, a theoretical study identified their microscopic difference- the ex situ approach characterizes the generated attosecond pulse, whereas the in situ approach resolves the underlying strong-field dynamics [23].

In this paper we study the scope of HHG spectroscopy resolving the internal probing mechanism in molecular systems. We focus on the measurements of $\mathrm{SF}_{6}, \mathrm{~N}_{2}$, and $\mathrm{CO}_{2}$, where intricate multichannel dynamics has been observed [13-16,19,24,25]. By applying the in situ and ex situ approaches in molecular systems, we perform an experimental demonstration of the conceptual microscopic difference between the two schemes. Next, we apply the in situ approach as a basic tool to probe the underlying pump-probe dynamics in molecular systems. By implementing the in situ measurement on ensembles of aligned molecules, we study the coupling between time and space, exploring the generality of HHG spectroscopy.

The in situ approach is based on the perturbation of the strong-field interaction by a weak SH field [21]. As the electron interacts with the perturbing field it accumulates a small complex phase shift [26] which serves as a temporal probe of the strong-field trajectories. Since the SH field breaks the symmetry between the positive and negative half cycles of the laser field, the accumulated phase leads to the appearance of even harmonics. By scanning the IR-SH delay, we modulate both even and odd harmonics. The modulation phase and contrast provide a direct insight into the underlying dynamics. Applying this approach in helium atoms we were able to isolate the tunneling dynamics from the recollision step, identifying its nonadiabatic nature [12].

Our experimental system consists of a $30-\mathrm{fs}, 800-\mathrm{nm}, 1-\mathrm{kHz}$ commercial laser system. The SH field, initially perpendicular to the fundamental field, is generated using 50- and $100-\mu \mathrm{m}$ 

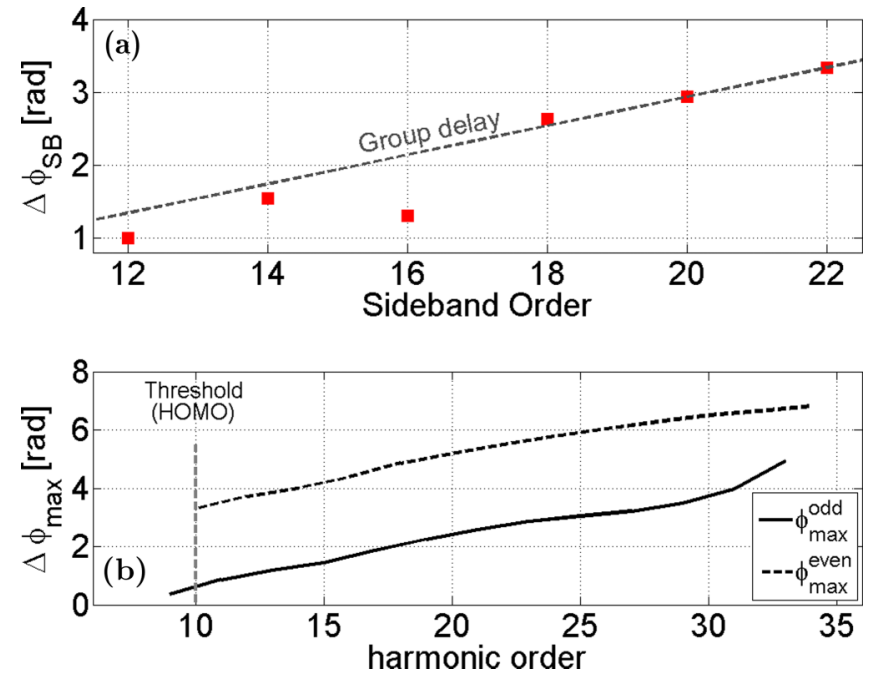

FIG. 1. (a) Sideband oscillations phase in a RABITT measurement in $\mathrm{SF}_{6}$ (red squares), compared with the expected linear harmonic phase difference for a single nonresonant channel (dashed gray). (b) Odd (solid lines) and even (dashed lines) harmonic oscillation phases in response to the two-color delay scan in $\mathrm{SF}_{6}$.

type-I beta barium borate (BBO) $\left(\beta \mathrm{BaB}_{2} \mathrm{O}_{4}\right)$ crystals. Parallel polarization is obtained using a $\lambda / 2$ zero-order waveplate for $800 \mathrm{~nm}$. Group-velocity dispersion is compensated using a birefringent crystal (calcite). The subcycle delay of the SH relative to the fundamental field is controlled using a pair of fused silica wedges. High harmonics are generated by focusing the two color beams into a pulsed $100-\mathrm{Hz}$ gas jet (Parker general valve) operating at 3-4 bars backing pressure. The harmonic spectrum is measured by an XUV spectrometer.

The first stage of our study explores the internal pumpprobe mechanism in $\mathrm{SF}_{6}$ molecules. Previous studies in $\mathrm{SF}_{6}$ identified the contributions of multiple channels and the significant role of shape resonances. Figure 1(a) describes an $e x$ situ measurement- the spectral phase resolved by a RABITT experiment [25]. A sharp modification of the spectral phase is clearly observed between the 15 th and the 17 th harmonics. This modification is very robust against variations of the laser intensity and macroscopic parameters such as gas pressure [27]. It has been assigned to a shape resonance in the radiative recombination process [25]. Figure 1(b) describes the in situ measurement, presenting the two-color oscillation phases in $\mathrm{SF}_{6}$. The phases change almost linearly with the harmonic number, exhibiting a nearly $\pi$ phase difference between odd and even harmonics. A similar response has been observed in a variety of atomic systems, in close agreement with the strong-field approximation (SFA) prediction [12]. The difference between the two measurements is striking-the sharp spectral feature between harmonics 15 and 17 observed in the RABITT measurement is completely absent in the in situ case.

In the following step we integrate the in situ measurement with molecular alignment to study the coupling between the spatial and the dynamical degrees of freedom. Experimentally, a pump pulse prepares an ensemble of aligned molecules. It is followed by a probe pulse at the half-revival delay, which is composed of the fundamental field and its $\mathrm{SH}$.
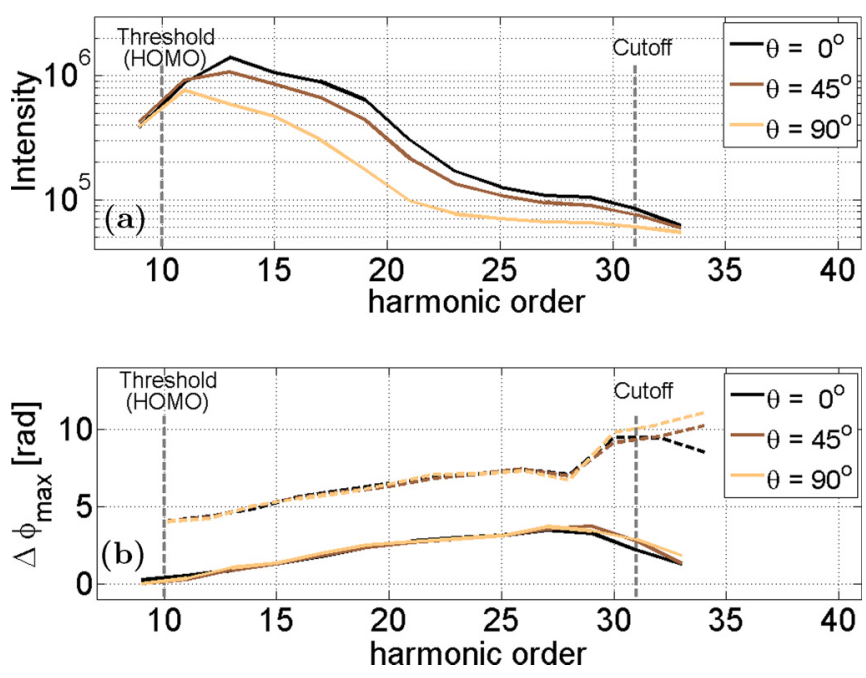

FIG. 2. (a) The HHG power spectrum of $\mathrm{N}_{2}$ for $0^{\circ}, 45^{\circ}$, and $90^{\circ}$ alignment angles. (b) Odd (solid lines) and even (dashed lines) harmonic oscillation phases in response to the two-color delay scan for $0^{\circ}, 45^{\circ}$, and $90^{\circ}$ alignment angles. Clearly, the rotation dramatically attenuates the power spectrum but introduces no significant change to the phases. The rapid change in the phase in the vicinity of the cutoff frequency is attributed to long trajectories.

Figure 2(a) describes the spectral intensity of the high harmonics, measured from $\mathrm{N}_{2}$ molecules, for different values of the alignment angle. A clear decrease of the harmonics intensity is observed as we rotate the molecule from $0^{\circ}$ (parallel to the laser polarization) to $90^{\circ}$ (perpendicular to the polarization). Previous ex situ studies measured the XUV spectral phase in $\mathrm{N}_{2}$ and observed a significant variation with the alignment angle as well [16]. Such dramatic structural dependence of the XUV spectrum has been extensively studied in $\mathrm{N}_{2}$ [6,28], reflecting the modification of the dipole moment with the molecular alignment angle.

In Fig. 2(b) we plot the phases of the high harmonics' oscillations for each alignment angle. Remarkably, the two-color response of the harmonics is independent of the alignment angle. The phases follow a trend similar to the one observed in $\mathrm{SF}_{6}$ - they change almost linearly with the harmonic number, exhibiting a nearly $\pi$ phase difference between odd and even harmonics for all alignment angles. Rotating the alignment angle leads to significant structural variations [Fig. 2(a)]; nevertheless, the dynamical properties as probed by the two-color measurement remain invariant [Fig. 2(b)]. The temporal-structural study of $\mathrm{N}_{2}$ establishes the most fundamental component in HHG spectroscopy-the basic pump-probe scheme is independent of the molecular structure.

What is the origin of the structural invariance and the conditions for its appearance? A deeper insight is obtained by the description of the dipole of the HHG process as a coherent sum of quantum trajectories [5]. This sum can be evaluated using the stationary phase approximation (SPA), defining the mapping between strong-field trajectories and harmonics [7]. Each harmonic order is described by a dominant trajectory defined by its ionization time $t_{0}^{s t}$, recollision time $t_{1}^{s t}$, and canonical momentum $\mathbf{p}^{s t}$. The structure of the molecule 
or atom is represented solely by the pre-exponential dipole terms. As long as the system does not exhibit any sharp structural features, the stationary points and therefore the internal dynamics are unaffected by the structure of the system.

Indeed, for a broad class of systems, the mapping between strong-field trajectories and harmonics is structurally independent. The ability to isolate this mapping and decouple it from the spatial degree of freedom poses an additional requirement-our in situ probe must be independent of the structural properties of the molecule. Under the SFA the ionization and recombination dipole moments can be evaluated at the stationary points as follows:

$$
\begin{aligned}
\mathbf{d}^{*}\left[\mathbf{p}^{s t}-\mathbf{A}\left(t_{1}^{s t}, \phi\right)\right] & =\mathbf{d}^{*}\left(\sqrt{2\left(N \omega-I_{p}\right)} \hat{\mathbf{n}}\right), \\
\mathbf{d}\left[\mathbf{p}^{s t}-\mathbf{A}\left(t_{0}^{s t}, \phi\right)\right] & =\mathbf{d}\left(i \sqrt{2 I_{p}} \hat{\mathbf{n}}\right)
\end{aligned}
$$

where $\omega$ is the fundamental laser frequency, $N$ is the harmonic order, $\phi$ is the two-color delay, $\mathbf{A}(t, \phi)$ is the vector potential, $\mathbf{d}[\mathbf{p}-\mathbf{A}(t, \phi)]$ is the dipole moment between the systems ground state and the corresponding continuum state at time $t$, and $\hat{\mathbf{n}}$ is a unit vector parallel to the polarization of the fundamental field. Clearly, according to Eq. (1), the functional dependency of the dipole moments at the stationary points can be replaced by a constant independent of the two-color delay, $\phi$. As long as we apply a parallel perturbation, we modify the dynamical properties of the interaction only via the strong-field trajectories, while the dipole moment itself remains unchanged.

Equation (1) has been derived under the SFA; however, the structural independence of our in situ scheme has been reproduced under more rigorous theoretical modeling as well [23]. Despite the many limitations of the SFA, such as Coulomb and multielectron effects [29-31], the model captures the structural independence observed in our experiments, providing physical insight into its origin. Note that the above analysis is valid for molecular ensembles that possess inversion symmetry.

The decoupling between the structural information and the internal dynamics clarifies the fundamental difference between the ex situ and in situ approaches. The RABITT measurement resolves the temporal shape of the emitted attosecond pulses. This measurement couples both strong-field dynamics and structural properties of the system. In contrast, the in situ measurement probes the dynamics of strong-field trajectories, identifies the role of the internal pump-probe process, and decouples it from the spatial degrees of freedom.

So far our analysis has considered a single ionization channel. Next, we consider the case of two noninteracting channels, initiated by the tunneling process. The in situ measurement independently perturbs each channel, leading to oscillations of the even harmonics according to

$$
\begin{aligned}
I^{N}(\theta, \phi)= & \mid A_{1}^{N}(\theta) \sin \left(\phi-\phi_{1}^{N}\right) \\
& +\left.e^{i \Delta I_{p} \tau} A_{2}^{N}(\theta) \sin \left(\phi-\phi_{2}^{N}\right)\right|^{2} .
\end{aligned}
$$

Here, $\Delta I_{p}$ is the difference between the ionization energies of the two channels and $\tau=t_{1}^{s t}-t_{0}^{s t}$ is the trajectory duration. $A_{1}^{N}(\theta)$ and $A_{2}^{N}(\theta)$ represent the complex contribution of each channel as a function of the alignment angle $\theta$, encoding the spatiotemporal properties of the hole wave function. The two phases $\phi_{1}^{N}$ and $\phi_{2}^{N}$ are the two-color oscillation phases
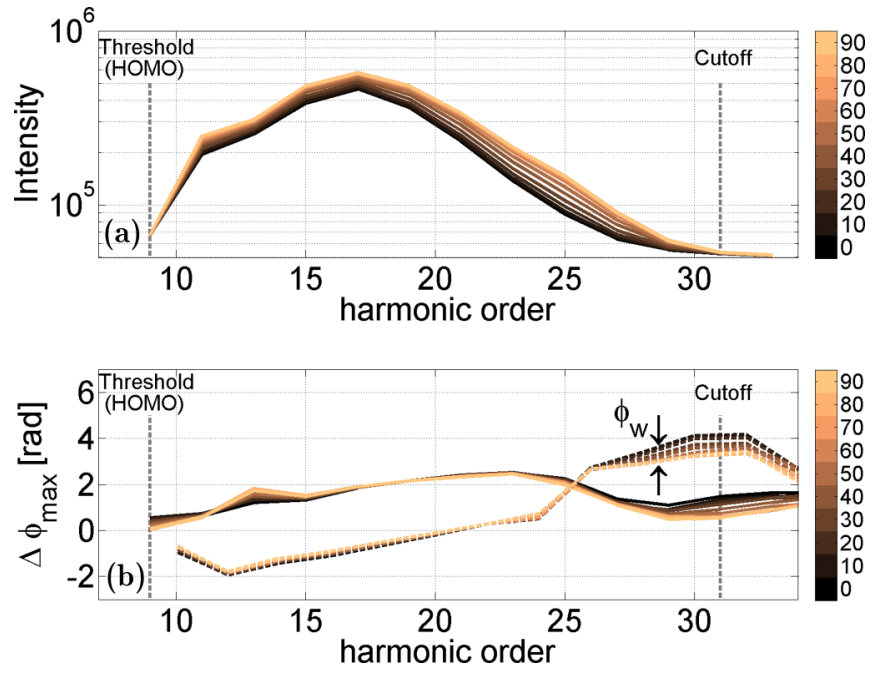

FIG. 3. (a) The HHG power spectrum of $\mathrm{CO}_{2}$ for various alignment angles from $0^{\circ}$ to $90^{\circ}$. (b) Odd (solid lines) and even (dashed lines) harmonic oscillation phases in response to the two-color delay scan for the same alignment angles. In $\mathrm{CO}_{2}$ the two-color phases vary monotonically with the alignment angle above harmonic $\mathrm{H} 25$.

associated with each channel, respectively. While we have considered two participating channels only, Eq. (2) can be easily extended to include any number of channels. By virtue of the in situ decoupling, the single-channel oscillation phases, $\phi_{1}^{N}, \phi_{2}^{N}$, are independent of the alignment angle. However, since both $A_{1}^{N}(\theta)$ and $A_{2}^{N}(\theta)$ are functions of the alignment angle $\theta$, the effective oscillation phase of the harmonic $I^{N}$ may vary as well. In this case the in situ measurements become extremely valuable, providing a clear fingerprint of the underlying multichannel dynamics.

We demonstrate the in situ measurement in the multichannel case in aligned $\mathrm{CO}_{2}$ molecules. HHG spectroscopy in $\mathrm{CO}_{2}$ molecules has been extensively studied in recent years [15,16,32-36]. In $\mathrm{CO}_{2}$, tunnel ionization evolves from multiple orbitals (channels) [19,24]. Rotating the alignment angle modifies the relative contribution of each channel and their coupling.

Figure 3 describes the dependence of the HHG intensity (a) and the two-color phases (b) on the alignment angle of $\mathrm{CO}_{2}$. The spectral intensity varies with the alignment angle, displaying the well-known attenuation in $0^{\circ}[15,36]$. Previous measurements preformed in similar conditions show a modification of the spectral phase with the alignment angle above harmonic $25[33,34]$. As in the $\mathrm{N}_{2}$ experiment, the two-color phase measured at harmonics 14-25 is almost invariant with the alignment angle, showing the same universal response. This response changes above harmonic 25, showing a significant monotonous variation of the phase with the alignment angle over a range of nine harmonic orders. Harmonic 13 shows a sharp alignment-dependent feature whose explanation lies beyond the scope of this paper.

Previous studies of the $\mathrm{CO}_{2}$ molecule under similar experimental conditions indicated that inner orbitals contribute to the HHG signal near the cutoff region [15]. Indeed, the two-color phases we measured for the lower harmonics completely 

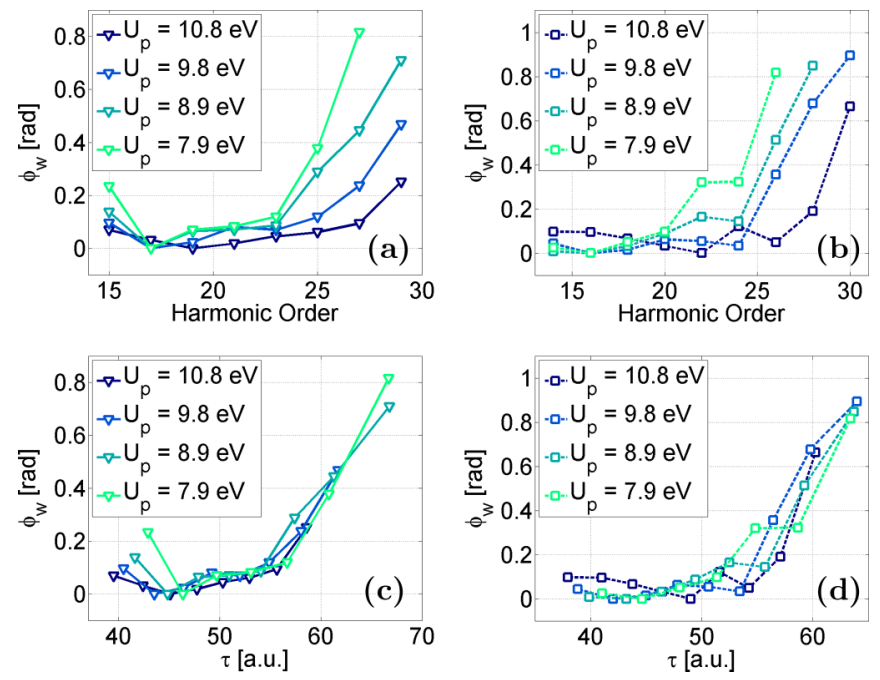

FIG. 4. The standard deviation of the two-color phase with respect to the alignment angle $\phi_{\mathrm{w}}$ for odd $(\mathrm{a}, \mathrm{c})$ and even $(\mathrm{b}, \mathrm{d})$ harmonics for various driving laser intensities. In the upper panel, $\phi_{\mathrm{w}}$ is presented against the harmonic axis, showing a divergence of the curves at different intensities. In the lower panel, the harmonic axis is replaced by the trajectory duration (in atomic units), $\tau=t_{1}^{s t}-t_{0}^{s t}$, resulting in coalescence of the measurements.

coincide, while the phases of harmonics in the vicinity of the cutoff show a strong angular dependence. This observation decouples the structural information from the dynamical one, separating between two spectral regions. It identifies the transition between a single orbital contribution, where only the highest occupied molecular orbital (HOMO) contributes, to a multiple-channel contribution, where hole dynamics is observed.

The angular dependence of the two-color phase encodes the coupling between hole dynamics and molecular structure. We quantify this by calculating for each harmonic the width of the distribution of the two-color phase with alignment angle, $\phi_{\mathrm{w}}$ [arrows in Fig. 3(b)]. For a single-channel response, the twocolor phase does not depend on the alignment angle, resulting in $\phi_{\mathrm{w}}$ values approaching the experimental noise level. A pronounced increase of $\phi_{\mathrm{w}}$ serves as a clear fingerprint of the transition between the single-channel and the multichannel case. Figures 4(a) and 4(b) describe the evaluated $\phi_{\mathrm{w}}$ for both odd and even harmonics at four different laser intensities. For all laser intensities, $\phi_{\mathrm{w}}$ approaches the noise level at the plateau harmonics while increasing dramatically close to the cutoff frequency. Such a response identifies the transition from a single to multiple-channel dynamics, providing an insight into the dynamical properties hidden in each harmonic.
Controlling the fundamental field's intensity gives a deeper insight into the strong-field interaction $[15,19,36]$. Figures 4 (a) and 4(b) show the modifications of $\phi_{\mathrm{w}}$ with the laser intensity. In order to study the origin of the intensity dependence we plot $\phi_{\mathrm{w}}$ as a function of the trajectory duration $\tau$. As we have established for the single-channel case, the mapping between harmonic number and $\tau$ is universal and structurally independent. The different orbitals that contribute to the HHG process have a relatively close $I_{p}$, and therefore we approximate $\tau$ to be associated with the HOMO orbital. Under our experimental conditions $\phi_{1}$ and $\phi_{2}$ are almost invariant with the intensity. Neglecting the influence of the laser intensity on the two channels' amplitudes, their relative phase, as described by Eq. (2), scales with $\tau$. Figures 4(c) and 4(d) present $\phi_{\mathrm{w}}$ as a function of $\tau$ for different laser intensities. As expected, in this analysis $\phi_{\mathrm{w}}(\tau)$ is almost invariant with the laser intensity. Indeed, the underlying dynamics scales with the fundamental parameter that defines each strong-field electron trajectory-its subcycle duration.

To conclude, in this paper we have studied the basic building block of HHG spectroscopy - the pump-probe measurement applied by the recolliding electron, in molecular systems. We have shown that the in situ approach decouples the pump-probe measurement represented by electron trajectories from the complex system under study. This picture remains valid as we advance from the single-channel to the multiple-channel case. While the hole dynamics is described via a complex multichannel interaction, the probing mechanism applied by the recolliding electron is universal and structurally independent.

Recent two-color HHG experiments in the condensed phase have shown that the strong-field dynamics could also be observed in solids [37]. Our work demonstrates that combining the two complimentary approaches-RABITT and in situ-will open new paths in the investigation of attosecond-scale phenomena in solids. Advancing to more complex systems, where coupling between the structural and dynamical properties can be expected, our scheme will become an essential component in advanced HHG spectroscopy.

N.D. is the incumbent of the Robin Chemers Neustein Professorial Chair, and gratefully acknowledges the Minerva Foundation, the Israeli Science Foundation, the European Research Council Starting Research Grant MIDAS, the Crown Photonics Center, and the I-Core Center for financial support. This project has received funding from the European Union's Horizon 2020 research and innovation programme under the Marie Sklodowska-Curie grant agreement No. 641272. Y.M. gratefully acknowledges the French National Research Agency (MISFTITS, ANR-14-CE32-0014).

G.O. and O.P. contributed equally to this work.
[1] M. Drescher, M. Hentschel, R. Kienberger, M. Uiberacker, V. Yakovlev, A. Scrinzi, T. Westerwalbesloh, U. Kleineberg, U. Heinzmann, and F. Krausz, Nature (London) 419, 803 (2002).

[2] R. Kienberger, E. Goulielmakis, M. Uiberacker, A. Baltuska, V. Yakovlev, F. Bammer, A. Scrinzi, T. Westerwalbesloh,
U. Kleineberg, U. Heinzmann et al., Nature (London) 427, 817 (2004).

[3] S. Baker, J. S. Robinson, C. Haworth, H. Teng, R. Smith, C. Chirilă, M. Lein, J. Tisch, and J. Marangos, Science 312, 424 (2006).

[4] P. B. Corkum, Phys. Rev. Lett. 71, 1994 (1993). 
[5] M. Lewenstein, P. Balcou, M. Y. Ivanov, A. L'Huillier, and P. B. Corkum, Phys. Rev. A 49, 2117 (1994).

[6] J. Itatani, J. Levesque, D. Zeidler, H. Niikura, H. Pépin, J.-C. Kieffer, P. B. Corkum, and D. M. Villeneuve, Nature (London) 432, 867 (2004).

[7] P. Salières, B. Carré, L. Le Déroff, F. Grasbon, G. Paulus, H. Walther, R. Kopold, W. Becker, D. Milošević, A. Sanpera et al., Science 292, 902 (2001).

[8] D. Shafir, H. Soifer, B. D. Bruner, M. Dagan, Y. Mairesse, S. Patchkovskii, M. Y. Ivanov, O. Smirnova, and N. Dudovich, Nature (London) 485, 343 (2012).

[9] H. Soifer, M. Dagan, D. Shafir, B. D. Bruner, M. Y. Ivanov, V. Serbinenko, I. Barth, O. Smirnova, and N. Dudovich, Chem. Phys. 414, 176 (2013).

[10] K. T. Kim, C. Zhang, A. D. Shiner, S. E. Kirkwood, E. Frumker, G. Gariepy, A. Naumov, D. Villeneuve, and P. Corkum, Nat. Phys. 9, 159 (2013).

[11] K. T. Kim, D. Villeneuve, and P. Corkum, Nat. Photonics 8, 187 (2014).

[12] O. Pedatzur, G. Orenstein, V. Serbinenko, H. Soifer, B. Bruner, A. Uzan, D. Brambila, A. Harvey, L. Torlina, F. Morales et al., Nat. Phys. 11, 815 (2015).

[13] Y. Mairesse, J. Higuet, N. Dudovich, D. Shafir, B. Fabre, E. Mével, E. Constant, S. Patchkovskii, Z. Walters, M. Y. Ivanov et al., Phys. Rev. Lett. 104, 213601 (2010).

[14] B. K. McFarland, J. P. Farrell, P. H. Bucksbaum, and M. Gühr, Science 322, 1232 (2008).

[15] O. Smirnova, Y. Mairesse, S. Patchkovskii, N. Dudovich, D. Villeneuve, P. Corkum, and M. Y. Ivanov, Nature (London) 460, 972 (2009).

[16] S. Haessler, J. Caillat, W. Boutu, C. Giovanetti-Teixeira, T. Ruchon, T. Auguste, Z. Diveki, P. Breger, A. Maquet, B. Carré et al., Nat. Phys. 6, 200 (2010).

[17] P. M. Kraus, B. Mignolet, D. Baykusheva, A. Rupenyan, L. Horný, E. F. Penka, G. Grassi, O. I. Tolstikhin, J. Schneider, F. Jensen et al., Science 350, 790 (2015).

[18] D. Faccialà, S. Pabst, B. D. Bruner, A. G. Ciriolo, S. De Silvestri, M. Devetta, M. Negro, H. Soifer, S. Stagira, N. Dudovich et al., Phys. Rev. Lett. 117, 093902 (2016).

[19] B. D. Bruner, Z. Mašín, M. Negro, F. Morales, D. Brambila, M. Devetta, D. Faccialà, A. G. Harvey, M. Ivanov, Y. Mairesse et al., Faraday Discuss. 194, 369 (2016).
[20] P. M. Paul, E. Toma, P. Breger, G. Mullot, F. Augé, P. Balcou, H. Muller, and P. Agostini, Science 292, 1689 (2001).

[21] N. Dudovich, O. Smirnova, J. Levesque, Y. Mairesse, M. Y. Ivanov, D. Villeneuve, and P. B. Corkum, Nat. Phys. 2, 781 (2006).

[22] J. M. Dahlström, T. Fordell, E. Mansten, T. Ruchon, M. Swoboda, K. Klünder, M. Gisselbrecht, A. L'Huillier, and J. Mauritsson, Phys. Rev. A 80, 033836 (2009).

[23] M. Spanner, J. B. Bertrand, and D. M. Villeneuve, Phys. Rev. A 94, 023825 (2016).

[24] L. Torlina, M. Ivanov, Z. B. Walters, and O. Smirnova, Phys. Rev. A 86, 043409 (2012).

[25] A. Ferré, A. Boguslavskiy, M. Dagan, V. Blanchet, B. Bruner, F. Burgy, A. Camper, D. Descamps, B. Fabre, N. Fedorov et al., Nat. Commun. 6, 5952 (2015).

[26] J. Dahlström, A. L'Huillier, and J. Mauritsson, J. Phys. B: At., Mol. Opt. Phys. 44, 095602 (2011).

[27] B. Manschwetus, N. Lin, J. Rothhardt, R. Guichard, T. Auguste, A. Camper, P. Breger, J. Caillat, M. Geleoc, T. Ruchon et al., J. Phys. Chem. A 119, 6111 (2015).

[28] I. V. Litvinyuk, K. F. Lee, P. W. Dooley, D. M. Rayner, D. M. Villeneuve, and P. B. Corkum, Phys. Rev. Lett. 90, 233003 (2003).

[29] S. Patchkovskii, Z. Zhao, T. Brabec, and D. M. Villeneuve, Phys. Rev. Lett. 97, 123003 (2006).

[30] S. Sukiasyan, C. McDonald, C. Destefani, M. Y. Ivanov, and T. Brabec, Phys. Rev. Lett. 102, 223002 (2009).

[31] V. P. Majety and A. Scrinzi, Phys. Rev. Lett. 115, 103002 (2015).

[32] T. Kanai, S. Minemoto, and H. Sakai, Nature (London) 435, 470 (2005).

[33] C. Vozzi, F. Calegari, E. Benedetti, J.-P. Caumes, G. Sansone, S. Stagira, M. Nisoli, R. Torres, E. Heesel, N. Kajumba et al., Phys. Rev. Lett. 95, 153902 (2005).

[34] W. Boutu, S. Haessler, H. Merdji, P. Breger, G. Waters, M. Stankiewicz, L. Frasinski, R. Taieb, J. Caillat, A. Maquet et al., Nat. Phys. 4, 545 (2008).

[35] X. Zhou, R. Lock, W. Li, N. Wagner, M. M. Murnane, and H. C. Kapteyn, Phys. Rev. Lett. 100, 073902 (2008).

[36] H. J. Wörner, J. B. Bertrand, P. Hockett, P. B. Corkum, and D. M. Villeneuve, Phys. Rev. Lett. 104, 233904 (2010).

[37] G. Vampa, T. Hammond, N. Thiré, B. Schmidt, F. Légaré, C. McDonald, T. Brabec, and P. Corkum, Nature (London) 522, 462 (2015). 